\title{
More Consistent Antimalarial Intake in First 5 Years of Disease Is Associated with Better Prognosis in Patients with Systemic Lupus Erythematosus
}

\author{
Rattapol Pakchotanon, Dafna D. Gladman, Jiandong Su, and Murray B. Urowitz
}

ABSTRACT. Objective. To examine whether more consistent use of antimalarial agents (AM) leads to better results in systemic lupus erythematosus (SLE).

Methods. From a longitudinal cohort study, we identified inception patients with a minimum of 5 years of followup. They were divided into 3 groups: patients who took AM $>60 \%$ of the time (group A), those who took $\mathrm{AM}<60 \%$ of the time (group B), and those who did not receive AM (group C) during the first 5 years of followup. Outcomes included increase in Systemic Lupus International Collaborating Clinics/American College of Rheumatology Damage Index (SDI), flare, achieving low disease activity (LDA), adjusted mean Systemic Lupus Erythematosus Disease Activity Index 2000, cumulative doses of steroids (CMS), and AM-related retinal toxicity. Regression analysis models were constructed to identify predictors of the outcomes.

Results. There were 459 patients identified: $236(51.4 \%)$ in group A, 88 (19.2\%) in group B, and 135 (29.4\%) in group C. The changes in SDI, flare event, and CMS were significantly lower in group A, which more often achieved LDA. Multivariable analysis revealed that the patients in group A had a lower risk of increasing SDI and were more likely to achieve LDA at Year 5 compared to the patients in group C. Patients taking AM had lower CMS over the 5 years of followup. There was only 1 patient with AM-related retinal toxicity in each group.

Conclusion. More consistent use of an AM over the first 5 years of SLE is associated with better outcomes. (First Release November 15 2017; J Rheumatol 2018;45:90-4; doi:10.3899/jrheum.170645)

Key Indexing Terms:

ANTIMALARIAL AGENT SYSTEMIC LUPUS ERYTHEMATOSUS DISEASE ACTIVITY

Antimalarial agents (AM), especially hydroxychloroquine (HCQ) and chloroquine (CQ), have demonstrated efficacy in systemic lupus erythematosus (SLE), mainly for constitutional symptoms (fever, fatigue, and weight loss) and mucocutaneous and musculoskeletal involvement ${ }^{1,2}$.

From the University of Toronto Lupus Clinic, Toronto Western Hospital, Centre for Prognosis Studies in the Rheumatic Diseases; University of Toronto, Toronto Western Research Institute, Toronto, Ontario, Canada. The University of Toronto Lupus Research Program is supported by the University Health Network, Lou and Marissa Rocca, and the Lupus Foundation of Ontario.

R. Pakchotanon, MD, Clinical Research Fellow, University of Toronto Lupus Clinic, Toronto Western Hospital, Centre for Prognosis Studies in the Rheumatic Diseases; D.D. Gladman, MD, FRCPC, Professor of Medicine, University of Toronto, Senior Scientist, Toronto Western Research Institute, and Co-Director, University of Toronto Lupus Clinic, Centre for Prognosis Studies in the Rheumatic Diseases, Toronto Western Hospital; J. Su, MSc, University of Toronto Lupus Clinic, Centre for Prognosis Studies in the Rheumatic Diseases, Toronto Western Hospital; M.B. Urowitz, MD, FRCPC, Professor of Medicine, University of Toronto, Senior Scientist, Toronto Western Research Institute, and Director, University of Toronto Lupus Clinic, Centre for Prognosis Studies in the Rheumatic Diseases.

Address correspondence to Dr. M.B. Urowitz, Centre for Prognosis Studies in the Rheumatic Diseases, Toronto Western Hospital, 399 Bathurst St., Toronto, Ontario M5T 2S8, Canada.E-mail:m.urowitz@utoronto.ca Accepted for publication September 1, 2017.
Moreover, AM have additional beneficial effects beyond controlling disease activity. AM have been shown to maintain disease remission ${ }^{2,3,4,5}$, prevent further damage ${ }^{6,7,8}$, protect against thrombosis ${ }^{9,10}$, and improve survival ${ }^{9,11,12}$. A systematic review performed mostly in patients with SLE found that the toxicities of AM were infrequent and mild, mainly gastrointestinal and cutaneous; cardiotoxicity was $\operatorname{rare}^{13}$. AM-related retinopathy in patients treated with HCQ and CQ was reported in only $0.1 \%$ and $2.5 \%$, respectively, after a mean duration of use of more than 10 years ${ }^{13}$. Because of their efficacy and low toxicity, current guidelines widely recommend the use of AM for all patients with SLE throughout the course of the disease unless toxicity ensues or there are contraindications ${ }^{14,15}$.

We aimed to examine the beneficial effect of AM treatment duration in the first 5 years of SLE on several longterm outcomes including damage accrual, flares, disease activity, steroid-sparing effect, and side effects, specifically AM-related retinopathy.

\section{MATERIALS AND METHODS}

Study population. The study was conducted at the University of Toronto Lupus Clinic, where patients have been followed prospectively since 1970 according to a standard protocol. Patients were included if they fulfilled 4

Personal non-commercial use only. The Journal of Rheumatology Copyright ( $)$ 2018. All rights reserved. 
or more of the American College of Rheumatology (ACR) classification criteria for $\mathrm{SLE}^{16}$, or 3 of the ACR criteria plus a biopsy compatible with SLE. Patient selection. An inception cohort of patients with SLE diagnosed within 1 year of presentation, seen between 1970 and 2015, was identified from the University of Toronto Lupus Clinic database. The inception SLE patients with at least 2 visits followed for a minimum of 5 years after the diagnosis of SLE were included in the current study. The patients were divided into 3 groups depending on the percentage of time that they took the AM during the 5 years of followup, as derived from their followup clinic visits. The duration of AM therapy was calculated based on patient-reported intake that was recorded in a standard protocol at every clinic visit. Patients who took $\mathrm{AM}>60 \%$ of the time formed group $\mathrm{A}$; patients who took $\mathrm{AM}<60 \%$ of the time formed group B; and patients who did not receive AM formed group C. In our center, CQ was prescribed at a dose $<3.5 \mathrm{mg} / \mathrm{kg}$ per day, and HCQ at a dose $<6.5 \mathrm{mg} / \mathrm{kg}$ per day.

Ethical review and approval was obtained from the University Health Network Research Ethics Board (REB number 11-0397-AE). Informed consent was collected from all patients at enrollment.

Clinical assessment. At enrollment and at 2 to 6 monthly intervals, patients were evaluated according to a standard protocol. Demographic data included sex, ethnicity, age at diagnosis, and calendar year of diagnosis. Disease activity was assessed by the Systemic Lupus Erythematosus Disease Activity Index 2000 (SLEDAI-2K) ${ }^{17}$, and damage was measured by Systemic Lupus International Collaborating Clinics/ACR Damage Index (SDI) ${ }^{18}$. Treatment variables included glucocorticosteroids (GCS), AM, and immunosuppressive drugs. All items necessary to complete the SLEDAI-2K and the SDI have been collected longitudinally in the protocol since its inception in 1970.

Outcome measures. Six outcomes were evaluated over 5 years: (1) organ damage was assessed yearly using the SDI; (2) flares were assessed at each visit and defined as any new clinical feature of the SLEDAI-2K since the previous assessment; (3) low disease activity (LDA) at Year 5 was defined by a clinical SLEDAI-2K score of $\leq 2$, not including serology ${ }^{19}$; (4) adjusted mean SLEDAI-2K over 5 years, a valid measure of the disease activity over time, was measured in the first 5 years; (5) cumulative doses of GCS were calculated; and (6) AM-related retinal toxicity was confirmed by an ophthalmologist.

Statistical analyses. Descriptive statistics for baseline information and outcomes were presented by mean $\pm \mathrm{SD}$ and $\mathrm{n}(\%)$. Differences among groups were compared by ANOVA and chi-square tests. Univariate and multivariable regression analyses were performed to identify the effect of the duration of AM treatment on the development of the 6 outcomes, controlling for patients' demographics, disease activity, and treatment. Logistic regression models for binary outcomes, Poisson regression models corrected for overdispersion by Pearson scaling for ordinal rare outcomes, and linear regression for continuous outcomes were constructed. For linear regression models, group effects were coded as 1 variable with values 1 (no treatment), 2 (inconsistent treatment), and 3 (more consistent treatment). The variable estimates reported are OR, relative risk (RR) and regression coefficient (ß), correspondingly. The statistical software SAS (version 9.3; SAS Institute) was used for all statistical analyses, and the significance level was set at 5\%.

\section{RESULTS}

Patient characteristics. A total of 777 inception patients with SLE were identified, of whom 459 with 5 years of followup were enrolled in our study. There were $236(51.4 \%)$ in group A, $88(19.2 \%)$ in group B, and 135 (29.4\%) in group C.

Demographic and disease-related features in the 3 groups are comparable, as shown in Table 1. Most of the patients were women and of white ethnicity. Mean \pm SD age at the diagnosis of SLE was $35.38 \pm 13.09$ years in group A, 34.42 \pm 14.45 years in group B, and $36.94 \pm 14.36$ years in group C. The SLE duration \pm SD at enrollment was $0.19 \pm 0.24$ years in group A, $0.24 \pm 0.30$ years in group B, and 0.25 \pm 0.29 years in group $\mathrm{C}$. At enrollment, SLEDAI-2K score (mean $\pm \mathrm{SD}$ ) was similar among the 3 groups: $9.67 \pm 8.00$ for group A, $10.51 \pm 7.26$ for group B, and $10.37 \pm 9.87$ for group C. However, renal-SLEDAI-2K is significantly lower in group A compared to groups B and C $(\mathrm{p}=0.004)$. SDI score at enrollment was comparable between groups (mean $\pm \mathrm{SD} ; 0.07 \pm 0.40$ in group $\mathrm{A}, 0.08 \pm 0.27$ in group $\mathrm{B}$, and $0.08 \pm 0.39$ in group $\mathrm{C}$ ). At enrollment, lupus nephritis manifestations were lower in group A than groups B and C, whereas musculoskeletal manifestations were less common in group C. The use of immunosuppressive drugs and GCS was comparable in the 3 groups. The patients diagnosed in earlier decades had a significantly lower percentage of AM use compared to the patients diagnosed in later decades (data not shown). HCQ is the most used AM (71.54\% in group A, and $61.11 \%$ in group $\mathrm{B})$. The mean $\pm \mathrm{SD}$ durations of antimalarial drug use over 5 years after enrollment were 4.7 \pm 0.8 year in group A, and $1.4 \pm 1.0$ year in group B.

The 6 outcomes among the 3 groups are shown in Table 2. The change in SDI, flare events, and cumulative doses of GCS were significantly lower in group A. In addition, patients in group A more often achieved a state of LDA compared to the other groups. AM-related retinal toxicity occurred in only 1 patient in group A and 1 in group B.

In multivariable analyses, adjusting for possible confounders including sex, ethnicity, age, disease duration, disease activity (SLEDAI-2K and renal-SLEDAI-2K), and treatment (model 1 in Table 3), the patients in group A had a lower risk of increasing SDI (RR 0.71, 95\% CI 0.52-0.95, $\mathrm{p}=0.02$ ) compared to the patients in group $\mathrm{C}$, while group $\mathrm{B}$ was not statistically different from group C. Other variables associated with a risk of increasing SDI were the age at SLE diagnosis (RR 1.02, 95\% CI 1.01-1.03, p < 0.00001) and cumulative dose of GCS (RR 1.01, 95\% CI 1.01-1.02, $\mathrm{p}=0.002)$. Moreover, the patients in group A were more likely to achieve LDA at Year 5 (OR 1.96, 95\% CI 1.18-3.26, $\mathrm{p}=0.009$ ) compared to the patients in group $\mathrm{C}$, while group $\mathrm{B}$ was not statistically different from group $\mathrm{C}$. The patients taking AM more consistently had a lower cumulative dose of GCS over the 5 years of followup ( $\beta-3.46,95 \%$ CI -4.65 to $-2.28, \mathrm{p}<0.0001)$, while group B was not statistically different from group $\mathrm{C}$. There was no association between the persistence of AM intake and the number of flares (RR $0.94,95 \%$ CI $0.82-1.09, \mathrm{p}=0.43)$ and adjusted mean SLEDAI-2K over 5 years ( $0.10,95 \%$ CI -0.19 to 0.40 , $\mathrm{p}=0.50$ ). An additional analysis, in which clinical manifestations at enrollment were added as potential confounders, demonstrated that patients in group A still had a lower risk of increasing SDI, more likely achieved LDA at Year 5, and had a lower cumulative dose of GCS over the 5 years of followup compared to the patients in group C (models 2 and 3 in Table 3). Decade of entry into the clinic was not significant in any of the models.

Personal non-commercial use only. The Journal of Rheumatology Copyright @ 2018 . All rights reserved. 
Table 1. Demographics and disease-related features at enrollment in the study population. Values are mean \pm SD or n (\%), unless otherwise specified.

\begin{tabular}{lccc}
\hline Variables & $\begin{array}{c}\text { Group A, AM Intake }> \\
60 \% \text { of Time, } \mathrm{n}=236\end{array}$ & $\begin{array}{c}\text { Group B, AM Intake }< \\
60 \% \text { of Time, } \mathrm{n}=88\end{array}$ & $\begin{array}{c}\text { Group C, Non-AM } \\
\text { Intake, } \mathrm{n}=135\end{array}$ \\
\hline Age at SLE diagnosis, yrs & $35.38 \pm 13.09$ & $34.42 \pm 14.45$ & $36.94 \pm 14.36$ \\
Age at enrollment, yrs & $35.57 \pm 13.06$ & $34.66 \pm 14.42$ & $37.19 \pm 14.34$ \\
Female & $210(89.0)$ & $79(89.8)$ & $115(85.2)$ \\
White/Black/Asian/others, $\%$ & $69.1 / 14 / 5.5 / 11.4$ & $67 / 17 / 6.8 / 9.1$ & $76.3 / 11.1 / 8.9 / 3.7$ \\
SLE duration at enrollment, yrs & $0.19 \pm 0.24$ & $0.24 \pm 0.30$ & $0.25 \pm 0.29$ \\
Clinical manifestation at enrollment & & & 0.47 \\
Lupus nephritis & $38(16.1)$ & $27(30.7)$ & $49(36.3)$ \\
$\quad$ Neuropsychiatric & $34(14.4)$ & $21(23.9)$ & $27(20.0)$ \\
Vasculitis & $30(12.7)$ & $12(13.6)$ & $11(8.1)$ \\
Mucocutaneous & $132(55.9)$ & $46(52.3)$ & $76(56.3)$ \\
Musculoskeletal & $71(30.1)$ & $19(21.6)$ & $16(11.9)$ \\
Serositis & $33(14.0)$ & $9(10.2)$ & $14(10.4)$ \\
Hematologic & $24(10.2)$ & $8(9.1)$ & $15(11.1)$ \\
Abnormal immunology & $161(68.2)$ & $54(61.4)$ & $83(61.5)$ \\
SLEDAI-2K score at enrollment & $9.67 \pm 8.00$ & $10.51 \pm 7.26$ & 0.11 \\
Renal-SLEDAI-2K score at enrollment & $1.54 \pm 4.01$ & $2.50 \pm 4.26$ & 0.37 \\
SDI score at enrollment & $0.07 \pm 0.40$ & $0.08 \pm 0.27$ & $3.02 \pm 4.54$ \\
Used glucocorticoids at enrollment & $126(53.4)$ & $52(59.1)$ & $0.08 \pm 0.39$ \\
Used immunosuppressive drugs at enrollment & $33(14.0)$ & $22(25.0)$ & $76(56.3)$ \\
\hline
\end{tabular}

AM: antimalarial agent; SDI: Systemic Lupus International Collaborating Clinics/American College of Rheumatology Damage Index; SLE: systemic lupus erythematosus; SLEDAI-2K: SLE Disease Activity Index 2000.

Table 2. Outcomes over 5 years after diagnosis of SLE in groups A, B, and C. Values are mean \pm SD or n (\%).

\begin{tabular}{lcccc}
\hline Outcomes & $\begin{array}{c}\text { Group A, } \\
\text { AM Intake }>60 \% \\
\text { of Time, } \mathrm{n}=236\end{array}$ & $\begin{array}{c}\text { Group B, } \\
\text { AM Intake }<60 \% \\
\text { of Time, } \mathrm{n}=88\end{array}$ & $\begin{array}{c}\text { Group C, } \\
\text { Non-AM intake, } \\
\mathrm{n}=135\end{array}$ & $\mathrm{p}$ \\
\hline Increase in SDI & $0.61 \pm 0.95$ & $0.85 \pm 1.30$ & $1.01 \pm 1.33$ & 0.003 \\
Flares & $2.66 \pm 1.96$ & $3.16 \pm 2.23$ & $3.23 \pm 2.79$ & 0.04 \\
LDA at yr 5 & $194(82.2)$ & $67(76.1)$ & $95(70.4)$ & 0.03 \\
Adjusted mean SLEDAI-2K & $4.36 \pm 2.90$ & $4.42 \pm 2.46$ & $4.83 \pm 3.74$ & 0.35 \\
Cumulative doses of GCS, g & $14.60 \pm 11.06$ & $20.22 \pm 13.35$ & $23.50 \pm 15.04$ & $<0.001$ \\
AM-related retinal toxicity & $1(0.4)$ & $1(1.1)$ & $0(0)$ & 0.45 \\
\hline
\end{tabular}

AM: antimalarial agent; GCS: glucocorticosteroids; LDA: low disease activity; SDI: Systemic Lupus International Collaborating Clinics/American College of Rheumatology Damage Index; SLE: systemic lupus erythematosus; SLEDAI-2K: SLE Disease Activity Index 2000.

\section{DISCUSSION}

The use of AM in SLE has increased over the decades. In our cohort of inception patients followed for a minimum of 5 years, we noted an increase of use from $36 \%$ in patients diagnosed prior to 1980 , to $53 \%$ in those diagnosed in the 1980 s, to $81 \%$ in those diagnosed in the 1990 s, to $87 \%$ in those diagnosed after 2000. This increase was likely influenced by the Canadian Hydroxychloroquine Study, which showed the protective role of AM in preventing flares ${ }^{3}$, as well as subsequent studies that demonstrated the beneficial effect of $\mathrm{AM}$ on thrombotic events ${ }^{9,10}$, damage accumulation $^{6,7,8}$, and mortality $9,11,12$. Moreover, Shinjo, et al demonstrated a protective effect of AM on mortality among SLE patients in a time-dependent manner ${ }^{12}$. The study showed that mortality rates (per 1000 person-months of followup) among users receiving AM for 6-11 months, 1-2 years, and $>2$ years were $3.85,2.7$, and 0.54 , respectively. In our study, we have addressed the question as to whether more consistent use of AM leads to better results than patients taking AM less consistently, in an inception cohort of patients with SLE over the first 5 years of disease.

Damage in SLE was associated with a $46 \%$ increased risk of future mortality ${ }^{7}$. The beneficial effect of antimalarial agents on cumulative damage in inception patients with SLE has been shown in previous studies. In the Systemic Lupus International Collaborating Clinics inception cohort of 1722 patients with SLE from diverse ethnic and geographic backgrounds, Bruce, et al demonstrated that patients with preexisting damage taking AM had lower rates of transition to higher damage ${ }^{7}$. Akhavan, et al showed in a nested 
Table 3. Multivariable regression analyses for outcomes at 5 years (group A vs group C).

\begin{tabular}{|c|c|c|c|c|c|c|c|c|c|c|}
\hline \multirow[t]{2}{*}{ Outcomes } & \multirow{2}{*}{$\begin{array}{l}\text { Statistic } \\
\text { Types }\end{array}$} & \multicolumn{3}{|c|}{ Model 1} & \multicolumn{3}{|c|}{ Model 2} & \multicolumn{3}{|c|}{ Model 3} \\
\hline & & PE & $95 \% \mathrm{CI}$ & $\mathrm{p}$ & PE & $95 \% \mathrm{CI}$ & $\mathrm{p}$ & PE & $95 \% \mathrm{CI}$ & $\mathrm{p}$ \\
\hline Increase in SDI & $\mathrm{RR}$ & 0.71 & $0.52-0.95$ & 0.02 & 0.72 & $0.53-0.98$ & 0.04 & 0.73 & $0.54-0.99$ & 0.04 \\
\hline LDA achievement at yr 5 & OR & 1.96 & $1.18-3.26$ & 0.009 & 2.09 & $1.25-3.52$ & 0.005 & 1.88 & $1.11-3.19$ & 0.02 \\
\hline $\begin{array}{l}\text { Adjusted mean SLEDAI-2K } \\
\text { over } 5 \text { yrs }\end{array}$ & $\beta$ & 0.10 & -0.19 to 0.40 & 0.49 & 0.12 & -0.18 to 0.42 & 0.45 & 0.11 & -0.20 to 0.42 & 0.49 \\
\hline
\end{tabular}

Group A: antimalarial agent intake $>60 \%$ of time; Group C: non-antimalarial agent intake. Model 1 is the primary analysis; Model 2 is the sensitivity analysis that forced musculoskeletal organ involvement into the model; Model 3 is the sensitivity analysis that forced renal organ involvement into the model. PE: parameter estimates; RR: relative risk from Poisson regression; OR from logistic regression; ß: regression coefficient from linear regression; GCS: glucocorticosteroids; LDA: low disease activity; SDI: Systemic Lupus International Collaborating Clinics/American College of Rheumatology Damage Index; SLEDAI-2K: Systemic Lupus Erythematosus Disease Activity Index 2000.

case-control study that the use of HCQ over the first 3 years of disease was significantly associated with less damage at 3 years after diagnosis when adjusted for disease activity and GCS dose, duration of disease, and calendar year of diagnosis (OR 0.34, 95\% CI 0.13-0.87) in 481 inception patients with SLE who did not have damage at baseline ${ }^{8}$. Our study shows that a more consistent intake of antimalarial therapy in the first 5 years of disease is associated with a reduced rate of damage accrual.

We found that the patients taking AM more consistently had a lower cumulative dose of GCS over the 5 years of followup. This finding was noted by Meinão, et al, who showed that $82 \%$ of SLE patients without life-threatening manifestations taking CQ $250 \mathrm{mg}$ daily could decrease prednisone by a minimum of $50 \%$ during 12 months of a randomized double-blind placebo-controlled trial, compared to $25 \%$ of patients with SLE who did not take CQ. This study also showed a significant reduction in disease exacerbations in patients using $\mathrm{CQ}^{4}$. Clowse, et al demonstrated the effect of HCQ in a prospective study of 257 pregnancies in women with SLE. In this study, they found that more women who discontinued HCQ during pregnancy took more prednisone. In addition, the average maximum daily dose of prednisone was lower among pregnant women who continued HCQ treatment than among those who did not. There was also a trend toward a lower flare rate in the HCQ treatment group compared to those who stopped taking $\mathrm{HCQ}^{20}$. Consistent with those previous reports, our results showed that patients who took AM more consistently more often achieved LDA at 5 years, and received a lower cumulative dose of GCS.

There are some limitations to our study. First, we calculated the duration of AM therapy based on patient-reported intake rather than by the Medication Adherence Self-report Inventory, pharmacy pill count, or antimalarial blood levels, which are more reliable measures of adherence to medication $21,22,23$. Second, our flare definition of any increase of SLEDAI-2K did not allow us to distinguish mild from severe flares. AM may have protected for severe flares.
Finally, our study design was a cohort study, which might be susceptible to selection bias. According to the clinical presentation at enrollment, patients who did not take AM had more lupus nephritis and less musculoskeletal involvement. However, we demonstrated that the adjusted mean SLEDAI-2K was comparable among the 3 groups at the enrollment. Nevertheless, in multivariable analysis the consistent use of AM treatment remained an independent predictor for less damage accrual, lower cumulative steroid dose, and achieving an LDA state, even after adjusting for the adjusted mean SLEDAI-2K, renal-SLEDAI-2K, and renal and musculoskeletal involvement.

We have shown that a more consistent intake of antimalarial therapy over the first 5 years of disease ( $>60 \%$ of the time) is associated with better outcomes. These findings highlight the importance of consistent intake or being prescribed AM early in the course of SLE to prevent adverse longterm outcomes.

\section{REFERENCES}

1. Rudnicki RD, Gresham GE, Rothfield NF. The efficacy of antimalarials in systemic lupus erythematosus. J Rheumatol 1975;2:323-30.

2. Williams HJ, Egger MJ, Singer JZ, Willkens RF, Kalunian KC, Clegg DO, et al. Comparison of hydroxychloroquine and placebo in the treatment of the arthropathy of mild systemic lupus erythematosus. J Rheumatol 1994;21:1457-62.

3. Canadian Hydroxychloroquine Study Group. A randomized study of the effect of withdrawing hydroxychloroquine sulfate in systemic lupus erythematosus. N Engl J Med 1991;324:150-4.

4. Meinão IM, Sato EI, Andrade LE, Ferraz MB, Atra E. Controlled trial with chloroquine diphosphate in systemic lupus erythematosus. Lupus 1996;5:237-41.

5. Tsakonas E, Joseph L, Esdaile JM, Choquette D, Senecal JL, Cividino A, et al. A long-term study of hydroxychloroquine withdrawal on exacerbations in systemic lupus erythematosus. The Canadian Hydroxychloroquine Study Group. Lupus 1998;7:80-5.

6. Pons-Estel GJ, Alarcon GS, Gonzalez LA, Zhang J, Vila LM, Reveille JD, et al. Possible protective effect of hydroxychloroquine on delaying the occurrence of integument damage in lupus: LXXI, data from a multiethnic cohort. Arthritis Care Res 2010;62:393-400.

Personal non-commercial use only. The Journal of Rheumatology Copyright @) 2018. All rights reserved. 
7. Bruce IN, O’Keeffe AG, Farewell V, Hanly JG, Manzi S, Su L, et al. Factors associated with damage accrual in patients with systemic lupus erythematosus: results from the Systemic Lupus International Collaborating Clinics (SLICC) Inception Cohort. Ann Rheum Dis 2015;74:1706-13.

8. Akhavan PS, Su J, Lou W, Gladman DD, Urowitz MB, Fortin PR. The early protective effect of hydroxychloroquine on the risk of cumulative damage in patients with systemic lupus erythematosus. J Rheumatol 2013;40:831-41.

9. Ruiz-Irastorza G, Egurbide MV, Pijoan JI, Garmendia M, Villar I, Martinez-Berriotxoa A, et al. Effect of antimalarials on thrombosis and survival in patients with systemic lupus erythematosus. Lupus 2006;15:577-83.

10. Mok CC, Tang SS, To CH, Petri M. Incidence and risk factors of thromboembolism in systemic lupus erythematosus: a comparison of three ethnic groups. Arthritis Rheum 2005;52:2774-82.

11. Alarcon GS, McGwin G, Bertoli AM, Fessler BJ, Calvo-Alen J, Bastian HM, et al. Effect of hydroxychloroquine on the survival of patients with systemic lupus erythematosus: data from LUMINA, a multiethnic US cohort (LUMINA L). Ann Rheum Dis 2007;66:1168-72.

12. Shinjo SK, Bonfa E, Wojdyla D, Borba EF, Ramirez LA, Scherbarth $\mathrm{HR}$, et al. Antimalarial treatment may have a time-dependent effect on lupus survival: data from a multinational Latin American inception cohort. Arthritis Rheum 2010;62:855-62.

13. Ruiz-Irastorza G, Ramos-Casals M, Brito-Zeron P, Khamashta MA. Clinical efficacy and side effects of antimalarials in systemic lupus erythematosus: a systematic review. Ann Rheum Dis 2010;69:20-8.

14. Tunnicliffe DJ, Singh-Grewal D, Kim S, Craig JC, Tong A. Diagnosis, monitoring, and treatment of systemic lupus erythematosus: a systematic review of clinical practice guidelines. Arthritis Care Res 2015;67:1440-52.
15. van Vollenhoven R, Voskuyl A, Bertsias G, Aranow C, Aringer M, Arnaud L, et al. A framework for remission in SLE: consensus findings from a large international task force on definitions of remission in SLE (DORIS). Ann Rheum Dis 2017;76:554-61.

16. Tan EM, Cohen AS, Fries JF, Masi AT, McShane DJ, Rothfield NF, et al. The 1982 revised criteria for the classification of systemic lupus erythematosus. Arthritis Rheum 1982;25:1271-7.

17. Gladman DD, Ibanez D, Urowitz MB. Systemic lupus erythematosus disease activity index 2000. J Rheumatol 2002;29:288-91.

18. Gladman D, Ginzler E, Goldsmith C, Fortin P, Liang M, Urowitz M et al. The development and initial validation of the Systemic Lupus International Collaborating Clinics/American College of Rheumatology damage index for systemic lupus erythematosus. Arthritis Rheum 1996;39:363-9.

19. Polachek A, Gladman DD, Su J, Urowitz MB. Defining low disease activity in systemic lupus erythematosus. Arthritis Care Res 2017;69:997-1003.

20. Clowse ME, Magder L, Witter F, Petri M. Hydroxychloroquine in lupus pregnancy. Arthritis Rheum 2006;54:3640-7.

21. Koneru S, Shishov M, Ware A, Farhey Y, Mongey AB, Graham TB, et al. Effectively measuring adherence to medications for systemic lupus erythematosus in a clinical setting. Arthritis Rheum 2007;57:1000-6.

22. Costedoat-Chalumeau N, Amoura Z, Hulot JS, Aymard G, Leroux G, Marra D, et al. Very low blood hydroxychloroquine concentration as an objective marker of poor adherence to treatment of systemic lupus erythematosus. Ann Rheum Dis 2007;66:821-4.

23. Durcan L, Clarke WA, Magder LS, Petri M. Hydroxychloroquine blood levels in systemic lupus erythematosus: clarifying dosing controversies and improving adherence. J Rheumatol 2015; 42:2092-7. 


\section{Correction}

More Consistent Antimalarial Intake in First 5 Years of Disease Is Associated with Better Prognosis in Patients with Systemic Lupus Erythematosus

Pakchotanon R, Gladman DD, Su J, Urowitz MB. More consistent antimalarial intake in first 5 years of disease is associated with better prognosis in patients with systemic lupus erythematosus. J Rheumatol 2018;45:90-4. The second affiliation of author Rattapol Pakchotanon should be Rheumatic Disease Unit, Department of Internal Medicine, Phramongkutklao Hospital and College of Medicine, Bangkok, Thailand.

doi:10.3899/jrheum.170645.C1 